\title{
(2) OPEN ACCESS \\ The prophylactic effects of long-acting granulocyte colony-stimulating factor for febrile neutropenia in newly diagnosed patients with epithelial ovarian cancer: a randomised controlled study
}

\author{
Lei Li, Shuiqing Ma $\mathbb{1}$, Ming Wu, Xianjie Tan, Sen Zhong, Jinghe Lang
}

- Additional material is published online only. To view please visit the journal online (http://dx.doi.org/10.1136/ bmjspcare-2019-001862).

Department of Obstetrics and Gynecology, Peking Union Medical College Hospital, Beijing, China

\section{Correspondence to}

Dr Shuiqing Ma, Obstetrics and Gynecology, Peking Union Medical College Hospital, Beijing 100730, China;

shuiqing_ma@163.com

Received 15 April 2019 Revised 18 July 2019 Accepted 21 August 2019 Published Online First 29 August 2019

\section{Check for updates}

(C) Author(s) (or their employer(s)) 2019. Re-use permitted under CC BY-NC. No commercial re-use. See rights and permissions. Published by BMJ.

To cite: Li L, Ma S, Wu M, et al. BMJ Supportive \& Palliative Care 2019;9:373-380.

\section{ABSTRACT}

Objective This study explored the prophylactic effects of long-acting granulocyte colonystimulating factor (G-CSF) for febrile neutropenia (FN) in newly diagnosed patients with epithelial ovarian cancer (EOC).

Methods Patients were randomised into a study group (long-acting G-CSF for all chemotherapy cycles) and a control group (short-acting G-CSF for first cycle and treatment per physician discretion for subsequent cycles) at a ratio of 1:2. The incidences of $\mathrm{FN}$ and myelosuppression and the number of clinical visits, medication doses, complete blood count $(C B C)$ tests and adverse events were compared between the two groups. A regression model was used to determine the risk factors for FN.

Results From 30 November 2018 to 1 April 2019,84 cases were included in the final analysis; there were 24 (28.6\%) and 60 (71.4\%) patients in the study and control groups, respectively, and 605 chemotherapy cycles. The study group or chemotherapy cycles utilising long-acting G-CSF had significantly fewer utilisations and doses of short-acting G-CSF; clinical visits; CBC tests; and incidences of FN and myelosuppression; and less G-CSFassociated pain. The utilisation of G-CSF was the only independent factor for FN in a binary regression model.

Conclusion Long-acting G-CSF could effectively reduce the incidences of $\mathrm{FN}$ and myelosuppression and had mild adverse effects in newly diagnosed patients with EOC receiving chemotherapy.

Trial registration number NCT03740464.

\section{INTRODUCTION}

To eliminate the burden of daily granulocyte colony-stimulating factor (G-CSF) injections, a long-acting pegylated recombinant human G-CSF (PEG-rhG-CSF; pegfilgrastim) has been developed to sustain relative stable concentration during neutropenia and decreases with neutrophil recovery. ${ }^{1}$ Pegfilgrastim has received marketing authorisation approval from many regions to reduce the incidence of chemotherapy-induced neutropenia because its efficacy and safety are similar to those of filgrastim with more convenience for use. ${ }^{2-6}$ A meta-analysis of randomised controlled trials (RCTs) ${ }^{7-9}$ and experience in real-world settings ${ }^{10}$ confirm the excellent success rates $(>50 \%)$ of filgrastim and pegfilgrastim for the primary prophylaxis of febrile neutropenia (FN). The efficiency and safety of these agents has been validated in older patients with cancer receiving chemotherapy in a community setting ${ }^{11}$ and in young adults and children with sarcoma. ${ }^{12}$ Hence, several guidelines recommend long-acting G-CSF as a firstline treatment for severe myelosuppression and/or FN. ${ }^{13-16}$ The choice of agent depends on convenience, cost and the clinical situation.

However, the effects of FN prevention have been infrequently evaluated in patients with gynaecological cancer. In a single-institution retrospective review of 46 patients with ovarian or primary peritoneal cancer who received prophylactic pegfilgrastim on the same day, no patient had FN episodes, hospitalisations or 
antibiotic use secondary to neutropenia nor did they have dose reductions or chemotherapy delays due to neutropenia. ${ }^{17}$ Several studies of solid tumours have also included patients with ovarian cancer. ${ }^{18-20}$ Despite a statement from multiple experts on gynaecological cancers regarding the application of prophylactic long-acting G-CSF, ${ }^{21}$ there is still very little evidence in terms of its efficiency and safety in gynaecological cancer. Additionally, in a study of Asian patients with lymphoma, pegfilgrastim prophylaxis did not show a therapeutic advantage in terms of preventing neutropenic outcomes compared with filgrastim prophylaxis. ${ }^{22}$

In this phase III RCT, we evaluated the prophylactic effects of PEG-rhG-CSF for FN in newly diagnosed patients with epithelial ovarian cancer (EOC) from a Chinese population.

\section{METHODS}

\section{Study design and ethical issues}

This is an RCT performed in a single teaching hospital. All eligible patients were randomised into a study group (long-acting G-CSF for all chemotherapy cycles) or a control group (long-acting G-CSF for the first cycle and medication per physician discretion for subsequent cycles) at a ratio of 1:2 according to the computer-generalised random numbers. The primary endpoint was the incidence of FN in each group. The secondary endpoints included the following: (1) clinical visits to the emergency room (ER), outpatient clinics and both locations; (2) the incidence of myelosuppression of all grades, grade $3 / 4$ and grade 4 and (3) adverse events associated with G-CSF.

\section{Patient enrolment and sample size}

All patients, who were newly diagnosed with EOC in the Gynecologic Oncology Unit of Peking Union Medical College Hospital from 30 November 2018 to 1 April 2019, were included. The inclusion criteria were as follows: each patient (1) had definitive pathological results of EOC; (2) had an Eastern Cooperative Oncology Group (ECOG) performance status score of $0-2$; (3) was aged 18 years or older; (4) received 3-4 weeks per cycle of platinum-based chemotherapy with or without debulking surgery; cases with weekly or biweekly chemotherapy regimens were not included; (5) was regularly followed up in the study centre and (6) provided consent for participation. The exclusion criteria were as follows: (1) failure to meet all the inclusion criteria; (2) non-compliance with the study protocols; (3) a history of chemotherapy or pelvic radiotherapy for malignancies; (4) the presence of immunosuppressive diseases such as organ transplantation or acquired immune deficiency syndrome; (5) treatment with weekly chemotherapy regimens and (6) the presence of haematological disorders.
The sample size was calculated according to the report by Balducci et al. ${ }^{18}$ Among patients receiving carboplatin and paclitaxel for ovarian or breast cancer, FN occurred in $2 \%$ and $6 \%$ of chemotherapy cycles, respectively, receiving pegfilgrastim. Thus, for the all-cycles arm and physician-discretion arm, ${ }^{18}$ at least 262 and 524 chemotherapy cycles were included in the study group and control group at a ratio of $1: 2$, which met the criteria for a class I probability (bilateral $\alpha)$ of 0.05 and power (1- $\beta$ ) of 0.80 by means of the Z-pooled approximate normal distribution method.

\section{Interventions}

All patients accepted platinum-based chemotherapy with or without primary or intermittent debulking surgeries. A regimen of paclitaxel $\left(175 \mathrm{mg} / \mathrm{m}^{2}\right)$ and carboplatin $(\mathrm{AUC}=5)$ of 3 weeks per cycle was used as the primary chemotherapy protocol for most patients. If the response was poor, the patient had a hypersensitive reaction, or there was another treatment purpose, an alternative regimen of 3-4 weeks per cycle was attempted. Surgeries included comprehensive staging for early stage disease and primary or intermittent debulking surgeries for late stage disease. Bevacizumab, an antiangiogenic inhibitor, was considered during treatment. For all patients, a complete blood count $(\mathrm{CBC})$ was requested for evaluation at a frequency of twice per week. At least one visit to an outpatient clinic was mandatory for all participants.

For the study group, $6 \mathrm{mg}$ of PEG-rhG-CSF (Qilu Pharmaceutical) was given to the patients 48 hours after chemotherapy. For the treatment of myelosuppression, short-acting G-CSF could be utilised.

For the control group, the treatment of myelosuppression was performed per physician discretion. However, during the first cycle, the physicians did not consider long-acting G-CSF. For subsequent cycles of chemotherapy, long-acting G-CSF was used if indicated by the physician.

\section{Measures}

A structured case report form was used to collect the epidemiological and clinical information of the patients via an electronic case system and the self-reports of the patients. The data for all the CBC tests, visits to the ER and/or outpatient clinics, and adverse events associated with the G-CSF treatment were extracted via the electronic case system and the means of reports collected from the patient if she was not registered in the system. Adverse events were graded according to the Common Terminology Criteria for Adverse Events V.4.03. ${ }^{23} \mathrm{FN}$ was defined as an oral temperature of $>38.3^{\circ} \mathrm{C}$ or two consecutive readings of $>38.0^{\circ} \mathrm{C}$ for 2 hours and an absolute neutrophil count (ANC) of $<0.5 \times 10^{9} / \mathrm{L}$ or as an ANC that was expected to fall below $0.5 \times 10^{9} / \mathrm{L}$. A visual analogue scale (VAS) score of $0-10$ was used to evaluate bone pain associated with G-CSF treatment. 


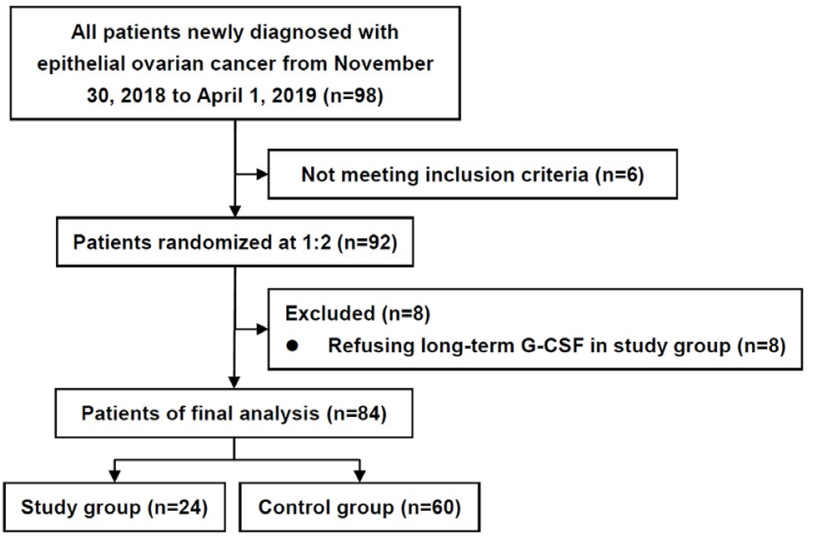

Figure 1 Flow diagram of the study. G-CSF, granulocyte colony-stimulating factor.

\section{Statistics}

Comparisons of the incidences of FN and myelosuppression between the study and control groups were conducted for each chemotherapy cycle according to the randomisation protocol (study and control groups) and according to the actual utilisation of long-acting G-CSF (chemotherapy cycles utilising long-acting and short-acting G-CSF). Comparisons of continuous variables were conducted with parametric methods if assumptions of normal distribution were confirmed. A binary regression model was used to estimate the HRs and 95\% CIs for the effects of long-acting G-CSF on FN and myelosuppression adjusted for age, body mass index, ECOG performance status, International Federation of Gynaecology and Obstetrics stage, history of radiotherapy, complications, chemotherapy regimen, use of bevacizumab and debulking surgery. Non-normally distributed variables and categorical data were compared by means of non-parametric tests. Unless otherwise stated, all analyses were performed with a two-sided significance level of 0.05 and conducted with SPSS V.23.0 software (SPSS, Chicago, IL, USA).

\section{RESULTS}

\section{Patients' characteristics}

The diagram of the study in presented in figure 1 . From 30 November 2018 to 1 April 2019, 98 patients were admitted for newly diagnosed EOC, and 84 cases were included for final analysis, with 24 (28.6\%) and $60(71.4 \%)$ patients in the study and control groups, respectively. Six patients were excluded: one patient had ECOG performance status score of 3, two patients received weekly paclitaxel treatment, two patients asked for treatment in other hospitals after the diagnosis and one patient refused further chemotherapy. The patients' characteristics are listed in table 1. More detailed data are provided in online supplementary file 1. Among all patients, 79 (94.0\%) underwent debulking surgeries, including 30 cases of primary surgeries and 49 of intermittent surgeries.
The total number of chemotherapy cycles was 605, and the median was 7 (range 4-11). Paclitaxel plus carboplatin was used in 557 cycles (92.1\%), and bevacizumab was used in 83 cycles $(13.7 \%)$. Other chemotherapy regimens included seven cycles of pegylated liposomal doxorubicin (PLD)/oxaliplatin, 35 cycles of $\mathrm{PLD} /$ carboplatin and 8 cycles of docetaxel/carboplatin. Three patients had a history of radiotherapy for breast cancer at least 1 year prior.

The study and control groups were well balanced in terms of most epidemiological and clinical characteristics (table 1). However, the study groups had fewer patients with diabetes and cerebrovascular disease and fewer cycles of bevacizumab use.

\section{FN and myelosuppression incidences}

In total, 33 patients experienced 38 incidences of $\mathrm{FN}$, with four patients in the study group (16.7\%) and 29 patients in the control group (48.3\%). After the incidence of FN, all these 33 patients received prophylactic long-acting (25 cases) or short-acting (eight cases) G-CSF. However, even after extensive prophylactic short-acting G-CSF treatment, five patients in the control group experienced two incidences of FN.

The FN and myelosuppression incidences per chemotherapy cycle are listed in table 2. All patients in the chemotherapy cycles of the study groups and in those utilising long-acting G-CSF had significantly fewer incidences of FN and myelosuppression of all grades, grade $3 / 4$ and grade 4 than the patients in the chemotherapy cycles of the control group and in those of the group utilising only short-acting G-CSF utilisation. However, the duration of $\mathrm{FN}$, admission times for FN and utilisation of antibiotics for FN showed no significant differences.

\section{Risk factors for FN}

In the chemotherapy cycles of the study and control groups, the FN incidences were 5/182 (2.7\%) and $33 / 483$ (7.8\%), respectively. In a binary regression model, after adjusting for the abovementioned risk factors, the randomisation of long-acting G-CSF was the only independent risk factor for FN (ORs 0.3, 95\% CI 0.1-0.8, $\mathrm{p}=0.019$ ).

In the chemotherapy cycles utilising long-acting and short-acting G-CSF, the FN incidences were 7/258 $(2.7 \%)$ and $31 / 347$ (8.9\%), respectively. The binary regression mode suggested that the utilisation of longacting G-CSF was the only independent risk factor for FN (ORs 0.2, 95\% CI 0.1-0.6, $\mathrm{p}=0.001$ ).

\section{Clinical visits}

In the study groups, 79/182 cycles (43.3\%) utilised short-acting G-CSF as a remedial treatment for myelosuppression, and the median doses of short-acting G-CSF were 2 (range 1-11). In the control groups, 230/423 cycles (54.4\%) utilised short-acting G-CSF 
Table 1 Epidemiologic characteristics of the patients

\begin{tabular}{|c|c|c|c|}
\hline & $\begin{array}{l}\text { Chemotherapy cycles in study } \\
\text { group }(n=182)\end{array}$ & $\begin{array}{l}\text { Chemotherapy cycles in control group } \\
(n=483)\end{array}$ & $P$ value \\
\hline Age (year), mean $\pm S D$ & $54.1 \pm 10.5$ & $52.9 \pm 7.1$ & 0.091 \\
\hline Body height $(\mathrm{m})$, mean $\pm S D$ & $58.6 \pm 6.9$ & $57.9 \pm 7.1$ & 0.286 \\
\hline Body weight $(\mathrm{kg})$, mean $\pm S D$ & $160.2 \pm 4.7$ & $160.8 \pm 4.5$ & 0.094 \\
\hline Body mass index $\left(\mathrm{kg} / \mathrm{m}^{2}\right)$, mean $\pm \mathrm{SD}$ & $22.8 \pm 2.4$ & $22.4 \pm 2.8$ & 0.064 \\
\hline ECOG performance status $n(\%)$ & & & 0.151 \\
\hline 0 & $105(57.7 \%)$ & $275(65.0 \%)$ & \\
\hline 1 & $54(29.7 \%)$ & $112(26.5 \%)$ & \\
\hline 2 & $23(12.6 \%)$ & $36(8.5 \%)$ & \\
\hline FIGO stage, $n(\%)$ & & & 0.274 \\
\hline I & $27(14.8 \%)$ & $48(11.3 \%)$ & \\
\hline$\|$ & $7(3.8 \%)$ & $16(3.8 \%)$ & \\
\hline III & $122(67.0 \%)$ & $273(64.5 \%)$ & \\
\hline IV & $26(14.3 \%)$ & $86(20.3 \%)$ & \\
\hline History of radiotherapy, n (\%) & $8(4.4 \%)$ & $16(3.8 \%)$ & 0.439 \\
\hline \multicolumn{4}{|l|}{ Complications, n (\%) } \\
\hline Hypertension & $34(18.7 \%)$ & $97(22.9 \%)$ & 0.244 \\
\hline Diabetes & $7(3.8 \%)$ & $35(8.3 \%)$ & 0.049 \\
\hline Cardiovascular disease & $0(0 \%)$ & $6(1.4 \%)$ & 0.186 \\
\hline Cerebrovascular disease & $0(0 \%)$ & $17(4.0 \%)$ & 0.006 \\
\hline DVT & $16(8.8 \%)$ & $36(8.5 \%)$ & 0.910 \\
\hline Chemotherapy cycles, mean \pm SD & & & 0.417 \\
\hline$\leq 6$ & $141(77.5 \%)$ & $340(80.4 \%)$ & \\
\hline$>6$ & $41(22.5 \%)$ & $83(19.6 \%)$ & \\
\hline Chemotherapy regimen, n (\%) & & & 0.074 \\
\hline PC & $173(95.1 \%)$ & $384(90.8 \%)$ & \\
\hline Others & $9(4.9 \%)$ & $39(9.2 \%)$ & \\
\hline Bevacizumab, n (\%) & $15(8.2 \%)$ & $68(16.1 \%)$ & 0.010 \\
\hline Debulking surgery, n (\%) & $24(13.2 \%)$ & $55(13.0 \%)$ & 0.951 \\
\hline
\end{tabular}

for myelosuppression, and the median dose of shortacting G-CSF was 2 (range 1-15).

The patients in the study group and in the longacting G-CSF utilisation group had significantly fewer visits to the $\mathrm{ER}$, outpatient clinics and both locations (table 2). The patients with long-acting G-CSF utilisation had significantly fewer CBC tests.

\section{Adverse events of G-CSF}

There appeared to be less pain associated with G-CSF in the long-acting G-CSF treatment group (table 2). However, the VAS score was not significantly different, with a mean VAS score less than 2 . The incidences of skin rash were similar in both groups, all of which were grade 1 . No other adverse events in terms of G-CSF were reported.

\section{DISCUSSION}

This randomised controlled study is the first RCT to evaluate the effects of G-CSF on the prevention of FN in patients with ovarian cancer. Treatment with G-CSF per physician discretion showed inferior outcomes for FN and myelosuppression. However, prophylactic treatment with short-acting G-CSF according to a stringent protocol would probably not result in such inferior outcomes. In a systematic review and meta-analysis, the weight of evidence from RCTs indicates little difference in efficacy between the shortacting and long-acting G-CSFs if dosed according to recommended guidelines. It was suggested long-acting G-CSFs in non-RCTs had greater efficacy, which may be a result of the underdosing of short-acting G-CSF in general practice in real-world usage. ${ }^{10}$ However, based on the findings of a systematic review of real-world comparative effectiveness studies, the risks of FN and FN-related complications are generally lower for prophylaxis with pegfilgrastim versus prophylaxis with short-acting G-CSF. ${ }^{8}$ Regarding patients with gynaecological cancer, filgrastim or pegfilgrastim was integrated in an NRG Oncology/Gynecologic Oncology Group study for the treatment of persistent or recurrent carcinoma of the uterine cervix, in which grade $3 / 4$ neutropenia occurred in 5 of 27 patients $(18.5 \%){ }^{24}$ 
Table 2 Prevalence, consequence and treatment of myelosuppression and FN in the study and control groups, and in long-acting and short-acting G-CSF users

\begin{tabular}{|c|c|c|c|c|c|c|}
\hline & $\begin{array}{l}\text { Chemotherapy } \\
\text { cycles in study } \\
\text { group }(\mathrm{n}=182)\end{array}$ & $\begin{array}{l}\text { Chemotherapy } \\
\text { cycles in control } \\
\text { group }(\mathrm{n}=483)\end{array}$ & P value & $\begin{array}{l}\text { Chemotherapy cycles } \\
\text { of long-acting G-CSF } \\
\text { utilisation }(n=258)\end{array}$ & $\begin{array}{l}\text { Chemotherapy cycles of } \\
\text { only short-acting G-CSF } \\
\text { utilisation }(n=347)\end{array}$ & P value \\
\hline Short-acting G-CSF utilisation, n (\%) & $79(43.4 \%)$ & $230(54.4 \%)$ & 0.013 & $95(36.8 \%)$ & $214(61.7 \%)$ & $<0.001$ \\
\hline Doses, mean \pm SD & $1.0 \pm 1.7$ & $2.0 \pm 2.6$ & $<0.001$ & $0.8 \pm 1.6$ & $2.3 \pm 2.7$ & $<0.001$ \\
\hline \multicolumn{7}{|l|}{ Clinical visits, mean $\pm S D$} \\
\hline Visits to ER & $0.3 \pm 0.9$ & $1.0 \pm 1.8$ & $<0.001$ & $0.5 \pm 1.2$ & $1.0 \pm 1.8$ & $<0.001$ \\
\hline Visits to outpatient clinics & $1.8 \pm 1.3$ & $2.5 \pm 1.4$ & $<0.001$ & $2.0 \pm 1.3$ & $2.5 \pm 1.5$ & $<0.001$ \\
\hline All visits & $2.1 \pm 1.7$ & $3.5 \pm 2.5$ & $<0.001$ & $2.5 \pm 1.9$ & $3.5 \pm 2.6$ & $<0.001$ \\
\hline$C B C$ times, mean $\pm S D$ & $5.0 \pm 1.9$ & $5.3 \pm 2.1$ & 0.192 & $4.9 \pm 2.0$ & $5.4 \pm 2.1$ & 0.003 \\
\hline $\mathrm{FN}, \mathrm{n}(\%)$ & $5(2.7 \%)$ & $33(7.8 \%)$ & 0.019 & $7(2.7 \%)$ & $31(8.9 \%)$ & 0.002 \\
\hline Lasting duration (day), mean \pm SD & $1.8 \pm 0.8(n=5)$ & $1.3 \pm 0.6(n=33)$ & 0.289 & $1.7 \pm 0.8(n=7)$ & $1.3 \pm 0.6(n=31)$ & 0.171 \\
\hline Admission for FN, n (\%) & $3 / 5(60 \%)$ & $22 / 33(66.7 \%)$ & 0.567 & $4 / 7(57.1 \%)$ & $21 / 31(67.7 \%)$ & 0.451 \\
\hline Antibiotics for FN, n (\%) & $5 / 5(100.0 \%)$ & $27 / 33(81.8 \%)$ & 0.401 & $7 / 7(100.0 \%)$ & $25 / 31(0.267 \%)$ & 0.267 \\
\hline \multicolumn{7}{|l|}{ Myelosuppression, n (\%) } \\
\hline All & $79(43.4 \%)$ & $292(69.0 \%)$ & $<0.001$ & $113(43.8 \%)$ & $258(74.4 \%)$ & $<0.001$ \\
\hline Grade 3/4 & $36(19.8 \%)$ & $174(41.1 \%)$ & $<0.001$ & $47(18.2 \%)$ & $163(47.0 \%)$ & $<0.001$ \\
\hline Grade 4 & $12(6.6 \%)$ & $73(17.3 \%)$ & $<0.001$ & $15(5.8 \%)$ & $70(20.2 \%)$ & $<0.001$ \\
\hline G-CSF-associated pain, n (\%) & $60 / 182(33.0 \%)$ & $200 / 423(47.3 \%)$ & 0.001 & $96 / 223(43.0 \%)$ & $164 / 221(74.2 \%)$ & $<0.001$ \\
\hline VAS, mean $\pm S D$ & $1.8 \pm 2.6$ & $1.4 \pm 2.0$ & 0.214 & $1.7 \pm 2.4$ & $1.4 \pm 2.0$ & 0.150 \\
\hline Skin rash, n (\%) & $4 / 182(2.2 \%)$ & $6 / 421(1.4 \%)$ & 0.355 & $5 / 258(1.9 \%)$ & $5 / 347(1.4 \%)$ & 0.434 \\
\hline Delay of chemotherapy, n (\%) & $17(9.3 \%)$ & $50(11.8 \%)$ & 0.373 & $23(8.9 \%)$ & $44(12.7 \%)$ & 0.144 \\
\hline Delayed days, mean $\pm S D$ & $0.5 \pm 2.2$ & $0.5 \pm 1.9$ & 0.930 & $0.4 \pm 2.0$ & $0.5 \pm 1.9$ & 0.162 \\
\hline
\end{tabular}

In a phase I study of patients with advanced ovarian cancer who were treated with biweekly dose-dense carboplatin/paclitaxel together with $6 \mathrm{mg}$ of pegfilgrastim on day 2 , FN (1/40, 2.5\%) was a dose-limiting toxicity that resulted in treatment discontinuation. ${ }^{25}$

Few reports have evaluated the prophylactic effects of G-CSF for the myelosuppression caused by newly marked targeted drugs. In our study, the addition of bevacizumab had no significant impact on the efficiency of long-acting G-CSF, which had recently been suggested by a study of patients with advanced colorectal cancer receiving chemotherapy and bevacizumab. ${ }^{26}$ The administration of pegfilgrastim was tolerable and did not negatively affect the tumour response or survival rate in this patient population. ${ }^{26}$

A number of studies have explored the administration timing and doses of pegfilgrastim. In our study, we prescribed long-acting G-CSF according to the instruction booklet with $6 \mathrm{mg}$ at 48 hours after chemotherapy. Such administration was reasonable according to a systematic review ${ }^{27}$ and consensus guidance recommendations. ${ }^{28}$ Patients who received pegfilgrastim on the day after chemotherapy had less severe and shorter suppression of the ANC than patients who received pegfilgrastim on the same day as chemotherapy. ${ }^{29}$ A prospective, multicenter GAIN study did not demonstrate that pegfilgrastim was more efficacious on day 4 than on day 2 with respect to grade 4 leucopenia, FN or infections. ${ }^{30}$ Pegfilgrastim injected as a single dose of either $100 \mu \mathrm{g} / \mathrm{kg}$ or as a total dose of $6 \mathrm{mg}$ (general approach) is considered an equally effective in terms of grade 3 neutropenia and above in patients with less intense chemotherapy. ${ }^{31}$ However, in this phase I study, there were no patients with gynaecological cancer. A multicenter study revealed that in patients with early breast cancer at high risk for FN, continued use of primary pegfilgrastim prophylaxis during all chemotherapy cycles is of clinical relevance and thus cannot be abandoned. ${ }^{32}$ However, G-CSF prophylaxis throughout all chemotherapy cycles is more costly than prophylaxis limited to the first two cycles. ${ }^{33}$ These controversies provide motivation for new studies to examine the most costeffective medication methods.

In our study, no severe adverse events in response to G-CSF occurred. Bone pain was the most common symptom, and several cases of skin rash occurred. No severe bone pain-related treatment-emergent adverse events or treatment-emergent adverse drug reactions were observed in patients with breast cancer receiving chemotherapy and G-CSF; the rates of bone painrelated events are similar between lipegfilgrastim and pegfilgrastim. ${ }^{34}$ Although rare, the physician should be aware of the possibility of interstitial pneumonia related to pegfilgrastim. ${ }^{35} \mathrm{~A}$ case of neutrophilic panniculitis and Sweet's syndrome lesions related to pegfilgrastim has been reported. ${ }^{36}$ Close monitoring and observation are needed for all patients receiving G-CSF treatment. 
The cost-effectiveness of long-acting G-CSF has been evaluated in several reports. An analysis found no evidence that the use of pegfilgrastim is associated with higher cost in patients with lymphoma and myeloma after high-dose chemotherapy and peripheral blood stem cell transplantation. ${ }^{37} \mathrm{~A}$ post hoc analysis in one RCT found that pegfilgrastim reduced the costs incurred for both the drugs and hospitalisations for adverse events as well as FN, although the total medical cost during chemotherapy increased. ${ }^{38}$ In our study, the utilisation of PEG-rhG-CSF not only reduced the usage of short-acting G-CSF and its dose but also significantly decreased the number of visits to the ER or outpatient clinics. The number of CBC tests in the long-acting G-CSF group was probably fewer than that of the tests in the short-acting G-CSF group. The expense of clinical visits, $\mathrm{CBC}$ tests and short-acting G-CSF doses saved by the utilisation of long-acting G-CSF will probably compensate for the high cost of the long-acting G-CSF. Other biosimilar pegfilgrastim products have been confirmed to have comparable efficacy and safety profiles to reference pegfilgrastim in a number of studies, ${ }^{939-42}$ and these products would provide more of a selection for patients.

There are several limitations in our study. The cost of the long-acting G-CSF limited the study to a 1:2 ratio for randomisation, which probably limited the power of the study. However, the enrolled patient numbers and the incidence of $\mathrm{FN}$ produced a power of 0.75 . Second, due to complications regarding the direct and indirect costs of diagnosis and treatment, we failed to construct an analysis model of costeffectiveness, even though long-acting G-CSF would greatly reduce the cost of medicine and the number of visits to clinics. Last, the effects of prophylactic shortacting and long-acting G-CSF were not compared in our study, which limited the generalisation of effects produced by long-acting G-CSF. Current guidelines recommended short-acting and long-acting G-CSF as prophylactic treatment for FN with similar effects. ${ }^{13} 14$ A randomised controlled study is needed to compare the real efficacy, adverse events and cost-effectiveness of various regimens of G-CSF.

\section{CONCLUSION}

In this phase III randomised controlled study, a longacting G-CSF, PEG-rhG-CSF, significantly reduced the incidences of $\mathrm{FN}$ and myelosuppression of all grades and grade 3/4. In addition, long-acting G-CSF decreased the number of clinical visits, the number of $\mathrm{CBC}$ tests and drug-associated bone pain despite the uniform follow-up protocol provided for all patients. The adverse events were mild and included pain and skin rash.

Contributors SM conceived of the original idea for the study, interpreted results, carried out the statistical analysis, edited the paper and was overall guarantor. LL obtained ethical approval, contributed to the preparation of the dataset, interpreted results and contributed to drafts of the paper. MW, XT, SZ and JL contributed to the study design, interpretation of results and commented on drafts of the paper.

Funding This study was supported by the Chinese Academy of Medical Sciences Initiative for Innovative Medicine (CAMS2017-I2M-1-002), and by the National Science-technology Support Plan Projects (2015BAI13B04).

Disclaimer The funders had no role in study design, data collection and analysis, decision to publish, or preparation of the manuscript.

Competing interests None declared.

Patient consent for publication Obtained.

Ethics approval The Institutional Review Board of Peking Union Medical College Hospital has approved this study (No. JS1723). The registration No. is NCT03740464 (clinicaltrials. gov). All patients provided consent before participation.

Provenance and peer review Not commissioned; externally peer reviewed.

Data availability statement All data relevant to the study are included in the article or uploaded as supplementary information.

Open access This is an open access article distributed in accordance with the Creative Commons Attribution Non Commercial (CC BY-NC 4.0) license, which permits others to distribute, remix, adapt, build upon this work noncommercially, and license their derivative works on different terms, provided the original work is properly cited, appropriate credit is given, any changes made indicated, and the use is noncommercial. See: http://creativecommons.org/licenses/by-nc/4. $0 /$.

\section{ORCID iD}

Shuiqing Ma http://orcid.org/0000-0003-2541-0025

\section{REFERENCES}

1 Geissler K, Gunzer M, Ostermann H. How safe is the administration of long-acting granulocyte colony-stimulating factor in cancer patients? Oncol Res Treat 2018;41:316-26.

2 Yang B-B, Savin MA, Green M. Prevention of chemotherapyinduced neutropenia with pegfilgrastim: pharmacokinetics and patient outcomes. Chemotherapy 2012;58:387-98.

3 Vogel CL, Wojtukiewicz MZ, Carroll RR, et al. First and subsequent cycle use of pegfilgrastim prevents febrile neutropenia in patients with breast cancer: a multicenter, double-blind, placebo-controlled phase III study. J Clin Oncol 2005;23:1178-84.

4 Vose JM, Crump M, Lazarus H, et al. Randomized, multicenter, open-label study of pegfilgrastim compared with daily filgrastim after chemotherapy for lymphoma. J Clin Oncol 2003;21:514-9.

5 Green MD, Koelbl H, Baselga J, et al. A randomized double-blind multicenter phase III study of fixed-dose single-administration pegfilgrastim versus daily filgrastim in patients receiving myelosuppressive chemotherapy. Ann Oncol 2003;14:29-35.

6 Holmes FA, O'Shaughnessy JA, Vukelja S, et al. Blinded, randomized, multicenter study to evaluate single administration pegfilgrastim once per cycle versus daily filgrastim as an adjunct to chemotherapy in patients with high-risk stage II or stage III/IV breast cancer. J Clin Oncol 2002;20:727-31.

7 Wang L, Baser O, Kutikova L, et al. The impact of primary prophylaxis with granulocyte colony-stimulating factors on febrile neutropenia during chemotherapy: a systematic review and meta-analysis of randomized controlled trials. Support Care Cancer 2015;23:3131-40. 
8 Mitchell S, Li X, Woods M, et al. Comparative effectiveness of granulocyte colony-stimulating factors to prevent febrile neutropenia and related complications in cancer patients in clinical practice: a systematic review. J Oncol Pharm Pract 2016;22:702-16.

9 Pfeil AM, Allcott K, Pettengell R, et al. Efficacy, effectiveness and safety of long-acting granulocyte colony-stimulating factors for prophylaxis of chemotherapy-induced neutropenia in patients with cancer: a systematic review. Support Care Cancer 2015;23:525-45.

10 Cornes P, Gascon P, Chan S, et al. Systematic review and meta-analysis of short- versus long-acting granulocyte colonystimulating factors for reduction of chemotherapy-induced febrile neutropenia. Adv Ther 2018;35:1816-29.

11 Flores IQ, Ershler W. Managing neutropenia in older patients with cancer receiving chemotherapy in a community setting. Clin J Oncol Nurs 2010;14:81-6.

12 Spunt SL, Irving H, Frost J, et al. Phase II, randomized, openlabel study of pegfilgrastim-supported VDC/IE chemotherapy in pediatric sarcoma patients. J Clin Oncol 2010;28:1329-36.

13 Klastersky J, de Naurois J, Rolston K, et al. Management of febrile neutropaenia: ESMO clinical practice guidelines. Ann Oncol 2016;27(suppl 5):v111-8.

14 NCCN Clinical Practice Guidelines in Oncology (NCCN Guidelines $\left.{ }^{\circledR}\right)$. Myeloid growth factor. version 1., 2018. Available: https://www.nccn.org/professionals/physician_gls/ pdf/mye

15 Smith TJ, Bohlke K, Lyman GH, et al. Recommendations for the use of WBC growth factors: American Society of clinical oncology clinical practice guideline update. J Clin Oncol 2015;33:3199-212.

16 Aapro MS, Bohlius J, Cameron DA, et al. 2010 update of EORTC guidelines for the use of granulocyte-colony stimulating factor to reduce the incidence of chemotherapyinduced febrile neutropenia in adult patients with lymphoproliferative disorders and solid tumours. Eur J Cancer 2011;47:8-32.

17 Schuman SI, Lambrou N, Robson K, et al. Pegfilgrastim dosing on same day as myelosuppressive chemotherapy for ovarian or primary peritoneal cancer. J Support Oncol 2009;7:225-8.

18 Balducci L, Al-Halawani $\mathrm{H}$, Charu V, et al. Elderly cancer patients receiving chemotherapy benefit from first-cycle pegfilgrastim. Oncologist 2007;12:1416-24.

19 Burris HA, Belani CP, Kaufman PA, et al. Pegfilgrastim on the same day versus next day of chemotherapy in patients with breast cancer, non-small-cell lung cancer, ovarian cancer, and non-Hodgkin's lymphoma: results of four multicenter, double-blind, randomized phase II studies. J Oncol Pract 2010;6:133-40.

20 Willis F, Woll P, Theti D, et al. Pegfilgrastim for peripheral CD34+ mobilization in patients with solid tumours. Bone Marrow Transplant 2009;43:927-34.

21 Petru E, Singer CF, Polterauer S, et al. Prophylactic long-acting granulocyte-colony stimulating factors (G-CSF) in gynecologic malignancies: an oncologic expert statement. Wien Med Wochenschr 2015;165:387-94.

22 Chan A, Leng XZ, Chiang JYL, et al. Comparison of daily filgrastim and pegfilgrastim to prevent febrile neutropenia in Asian lymphoma patients. Asia Pac J Clin Oncol 2011;7:75-81.

23 Common terminology criteria for adverse events (CTCAE) v4.03. National Cancer Institute. Available: https://ctep.cancer. gov/protocolDevelopment/electronic_applications/ctc.htm\# ctc_50

24 Kunos C, Deng W, Dawson D, et al. A phase I-II evaluation of veliparib (NSC \#737664), topotecan, and filgrastim or pegfilgrastim in the treatment of persistent or recurrent carcinoma of the uterine cervix: an NRG Oncology/ Gynecologic Oncology Group study. Int J Gynecol Cancer 2015;25:484-92.
25 Tiersten AD, Sill MW, Knight D, et al. A phase I trial of dosedense (biweekly) carboplatin combined with paclitaxel and pegfilgrastim: a feasibility study in patients with untreated stage III and IV ovarian, tubal or primary peritoneal cancer: a gynecologic Oncology Group study. Gynecol Oncol 2010;118:303-7.

26 Pinter T, Klippel Z, Cesas A, et al. A phase III, randomized, double-blind, placebo-controlled trial of pegfilgrastim in patients receiving first-line FOLFOX/Bevacizumab or FOLFIRI/Bevacizumab for locally advanced or metastatic colorectal cancer: final results of the pegfilgrastim and anti-VEGF evaluation study (paves). Clin Colorectal Cancer 2017;16:103-14.

27 Lyman GH, Allcott K, Garcia J, et al. The effectiveness and safety of same-day versus next-day administration of long-acting granulocyte colony-stimulating factors for the prophylaxis of chemotherapy-induced neutropenia: a systematic review. Support Care Cancer 2017;25:2619-29.

28 Aapro M, Boccia R, Leonard R, et al. Refining the role of pegfilgrastim (a long-acting G-CSF) for prevention of chemotherapy-induced febrile neutropenia: consensus guidance recommendations. Support Care Cancer 2017;25:3295-304.

29 Li Y, Klippel Z, Shih X, et al. Trajectory of absolute neutrophil counts in patients treated with pegfilgrastim on the day of chemotherapy versus the day after chemotherapy. Cancer Chemother Pharmacol 2016;77:703-12.

30 Loibl S, Mueller V, von Minckwitz G, et al. Comparison of pegfilgrastim on day 2 vs. day 4 as primary prophylaxis of intense dose-dense chemotherapy in patients with nodepositive primary breast cancer within the prospective, multi-center gain study: (GBG 33). Support Care Cancer 2011;19:1789-95.

31 Qin Y, Han X, Wang L, et al. A phase I study of different doses and frequencies of pegylated recombinant human granulocytecolony stimulating factor (PEG rhG-CSF) in patients with standard-dose chemotherapy-induced neutropenia. Chin J Cancer Res 2017;29:402-10.

32 Aarts MJ, Peters FP, Mandigers CM, et al. Primary granulocyte colony-stimulating factor prophylaxis during the first two cycles only or throughout all chemotherapy cycles in patients with breast cancer at risk for febrile neutropenia. J Clin Oncol 2013;31:4290-6.

33 Aarts MJ, Grutters JP, Peters FP, et al. Cost effectiveness of primary pegfilgrastim prophylaxis in patients with breast cancer at risk of febrile neutropenia. J Clin Oncol 2013;31:4283-9.

34 Bondarenko IM, Bias P, Buchner A. Incidence of bone pain in patients with breast cancer treated with lipegfilgrastim or pegfilgrastim: an integrated analysis from phase II and III studies. Support Care Cancer 2016;24:267-73.

35 Shirasawa M, Nakahara Y, Niwa $\mathrm{H}$, et al. Interstitial pneumonia following administration of pegfilgrastim during carboplatin and etoposide chemotherapy for small-cell lung cancer. Mol Clin Oncol 2016;5:714-6.

36 Llamas-Velasco M, García-Martín P, Sánchez-Pérez J, et al. Sweet's syndrome with subcutaneous involvement associated with pegfilgrastim treatment: first reported case. J Cutan Pathol 2013;40:46-9.

37 Perrier L, Lefranc A, Pérol D, et al. Cost effectiveness of pegfilgrastim versus filgrastim after high-dose chemotherapy and autologous stem cell transplantation in patients with lymphoma and myeloma: an economic evaluation of the palm trial. Appl Health Econ Health Policy 2013;11:129-38.

38 Miyake O, Murata K, Tanaka S, et al. Costs associated with febrile neutropenia in Japanese patients with primary breast cancer: post-hoc analysis of a randomized clinical trial. Jpn J Clin Oncol 2018;48:410-6. 
39 Blackwell K, Gascon P, Jones CM, et al. Pooled analysis of two randomized, double-blind trials comparing proposed biosimilar LA-EP2006 with reference pegfilgrastim in breast cancer. Ann Oncol 2017;28:2272-7.

40 Zhou C, Huang Y, Wang D, et al. A randomized multicenter phase III study of single administration of Mecapegfilgrastim (HHPG-19K), a pegfilgrastim Biosimilar, for prophylaxis of chemotherapy-induced neutropenia in patients with advanced non-small-cell lung cancer (NSCLC). Clin Lung Cancer 2016;17:119-27.
41 Volovat C, Bondarenko I, Gladkov O, et al. Efficacy and safety of lipegfilgrastim compared with placebo in patients with non-small cell lung cancer receiving chemotherapy: post hoc analysis of elderly versus younger patients. Support Care Cancer 2016;24:4913-20.

42 Bondarenko I, Gladkov OA, Elsaesser R, et al. Efficacy and safety of lipegfilgrastim versus pegfilgrastim: a randomized, multicenter, active-control phase 3 trial in patients with breast cancer receiving doxorubicin/docetaxel chemotherapy. BMC Cancer 2013;13:386. 